\title{
Therapeutic strategies utilization and resource consumption in patients treated for psoriatic arthritis: findings from a real-world analysis in an Italian setting
}

This article was published in the following Dove Press journal:

Patient Preference and Adherence

\author{
L Degli Esposti ${ }^{1}$ \\ $\checkmark$ Perrone' \\ D Sangiorgi' \\ D Alessandrini' \\ S Buda' \\ F Cantini ${ }^{2}$ \\ E Mazzini ${ }^{3}$ \\ C Toma ${ }^{3}$ \\ F De Solda ${ }^{3}$ \\ 'CliCon S.r.l. Health, Economics \& \\ Outcomes Research, Ravenna, Italy; \\ ${ }^{2}$ Division of Rheumatology, Hospital \\ of Prato, Azienda USL Toscana Centro, \\ Prato, Italy; ${ }^{3}$ Bristol-Myers Squibb, \\ Roma, Italy
}

\begin{abstract}
Purpose: The purpose of this study was to analyze the therapeutic strategies and estimate the health care resource consumption in patients with psoriatic arthritis (PsA).

Patients and methods: An observational retrospective cohort analysis of administrative databases of six Italian Local Health Units was performed. Patients $\geq 18$ years with a hospitalization discharge diagnosis of PsA (International Classification of Diseases, Ninth Revision code: 696.0) or exemption code (045.696.0) for PsA from January 1, 2010 to December 31, 2015 (inclusion period), with at least one prescription of any therapy used for PsA were included. The index date (ID) was the first date matching with at least one of the inclusion criteria during the inclusion period. All patients were followed up after the ID until the end of data availability. Baseline C-reactive protein (CRP) levels ( \pm 6 months in relation to the ID) were also analyzed. Results: A total of 2,408 (prevalence 0.83 per 1,000) patients with PsA (male 52\%; median age 54 years) were included in the study; patients were already treated for PsA in $42.4 \%$ of cases. At 1 year of follow-up, $73 \%$ of the patients received one systemic drug, while $22 \%$ of patients received two systemic drugs; in addition, our results show an increase in the number of add-on or switches in a longer follow-up period. The utilization of biologic agents was higher among patients with previous PsA treatment, showing a progression of the pathology. Overall, a medium/high level of CRP at baseline was observed among more than half of the overall sample, with slight changes across subgroups in analysis. The average health care costs were $€ 1,966.4$ and $€ 13,914$ per year for patients treated with conventional systemic therapy and biological agents, respectively. Conclusion: A better knowledge of prescription therapeutic scheme and economic burden of PsA could stimulate the rational development of health programs aimed at potentiating services for its management.
\end{abstract}

Keywords: psoriatic arthritis, drug utilization, real-world data, treatment patterns

\section{Introduction}

Psoriatic arthritis (PsA) is a chronic inflammatory disease of the musculoskeletal system associated with psoriasis characterized by variable clinical symptoms with very heterogeneous degrees of disease suffering for patients. ${ }^{1}$ The exact incidence and prevalence of PsA worldwide are rather undefined and its estimation has been difficult with a substantial variability in the incidence and prevalence of PsA by country. . $^{2,3}$ In the Italian population, the estimated prevalence of PsA is $0.42 \%{ }^{4}$

PsA is a multifaceted disease with inflammatory involvement of skin and nails, peripheral joints, entheses, tendons, and axial skeleton. This variability necessitates
Correspondence: L Degli Esposti CliCon S.r.l. Health, Economics \& Outcomes Research, Via Salara, 36, 48100 Ravenna, Italy

Tel +3954 438393

Fax+3954421 2699

Email degliesposti@clicon.it
Patient Preference and Adherence 2019:13 187-194

187

Dovepress f $y$ in 0

http:

(c) (1) (9) 2019 Degli Esposti et al This work is published and licensed by Dove Medical Press Limited. The full terms of this license are available at https://www.dovepress.com/terms.php (c)
hereby accept the Terms. Non-commercial uses of the work are permitted without any further permission from Dove Medical Press Limited, provided the work is properly attributed. For permission hereby accept the Terms. Non-commercial uses of the work are permitted without any further permission from Dove Medical Press Limited, provided the work is properly attributed. For permission
for commercial use of this work please see paragraphs 4.2 and 5 of our Terms (htpps//wwww.dovepress.com/terms php). 
an individualized therapy of patients with different therapy targets. ${ }^{5-7}$ Patients with PsA may also have an increased risk of comorbidity conditions, especially cardiovascular disease, compared with the general population.

Treatments for PsA can relieve pain, reduce swelling, help to keep joints working properly, and possibly prevent further joint damage. The therapeutic choice depends on the clinical history of the patient, on the joint involvement, and on the tolerability of the different available drugs. Several therapeutic options are available for patients with PsA, including nonsteroidal anti-inflammatory drugs (NSAIDs), glucocorticoids, traditional disease-modifying anti-rheumatic drugs (also called conventional synthetic disease-modifying antirheumatic drugs [csDMARDs]), systemic biologic (also called biologic disease-modifying anti-rheumatic drug [bDMARDs]) therapies (anti-tumor necrosis factor [TNF- $\alpha$ ], anti-IL 12/23, anti-IL/17), and targeted synthetic DMARD (also called targeted synthetic disease-modifying anti-rheumatic drug [tsDMARD]) which is a phosphodiesterase 4 (PDE4) inhibitor. ${ }^{5,8}$

In 2012, European League against Rheumatism (EULAR) recommendations for the pharmacological treatment of PsA were published. ${ }^{9}$ Since then, new treatment strategies and importantly, drugs with new mechanisms of action (such as bDMARDs targeting IL-12/23/17 pathways and tsDMARD [PDE4 inhibitor]) have been approved for and/or evaluated in PsA. In 2015, an EULAR Task Force updated the PsA management recommendations indicating csDMARDs as an initial therapy after failure of NSAIDs and local therapy for active disease, followed, if necessary, by a bDMARD or a tsDMARD. ${ }^{8}$

PsA imposes a substantial economic burden to the patients and the society due to direct and indirect costs. ${ }^{10}$ The clinical burden of PsA contributes to direct medical costs. Indirect costs, including productivity loss and disability caused by limitations in functioning and activities of daily living, also contribute to the total costs of PsA. Although the burden of psoriasis has been described extensively in the literature, the burden of illness associated with PsA in Italy has not been precisely estimated.

The purpose of the present study was to analyze the therapeutic strategies and to estimate the health care resource consumption in patients with PsA.

\section{Patients and methods Data source}

This study was conducted using administrative database and laboratory test values database from a sample of six Italian
Local Health Units (LHUs). The LHUs database used for this study includes around two million inhabitants $3.33 \%$ of the national population) located in northern, central, and southern Italy. In particular, the following databases were used: the Beneficiaries' database, which contains all demographic data for patients in analysis (deaths included); the Pharmaceuticals Prescription database, which contains all drug supplies for patients in analysis; the Hospital Discharge database, which contains all hospitalizations data for patients in analysis; the Outpatient Specialist Services database, which contains all information about diagnostic tests and visits for patients in analysis; the Exemption Ticket for pathology database, which contains all information about the attributed exemption code, which permit to receive drugs free of charge, and the Laboratory test values database (for four out of the six LHUs involved), which contains laboratory results for patients in analysis.

The patient code in each database allowed electronic linkage between all different databases. To guarantee patients' privacy, an anonymous univocal numeric code was assigned to each subject included in the study. No identifiers related to patients were provided to the authors. All results have been produced in an aggregate way. According to the Italian Guidelines regarding the conduction of observational studies, ${ }^{11}$ established by the Italian Drug Agency, "Agenzia Italiana del Farmaco - AIFA" on March 20, 2008, this study has been notified to the local Ethics Committee of all participating LHUs and each participating LHU has approved the study. Informed consent was not obtained, since it is not required when using encrypted retrospective information for research purposes.

\section{Cohort definition}

An observational retrospective cohort analysis was performed. Adult patients ( $\geq 18$ years) with a hospitalization discharge diagnosis of PsA (classified according to the International Classification of Diseases, Ninth Revision [code: 696.0]) or exemption code (045.696.0) for PsA from January 1, 2010 to December 31, 2015 (inclusion period), with at least one prescription of any therapy used for PsA were included.

The first date matching with at least one of the inclusion criteria during the considered period was selected as the index date (ID). All patients were followed up after the ID until the end of data availability, date of death, or exiting the database (follow-up period). Patients who were transferred to another LHU during the follow-up period were excluded from the analysis. 


\section{Study variables}

Data on baseline characteristics, including demographics (age and gender), medical history (hospital admission, prescribed drugs, and profile of comorbidity) were collected. The PsA treatments of interest were all systemic nonbiologic therapies (also called "csDMARDs": methotrexate [ATC code: L04AX03], cyclosporine [ATC code: L04AD01], sulphasalazine [ATC code: A07EC01], leflunomide [ATC code: L04AA13], hydroxychloroquine [ATC code: P01BA02]) and all systemic biologic therapies available at the time of the analysis (infliximab [ATC code: L04AB02], etanercept [ATC code: L04AB01], adalimumab [ATC code: L04AB04], certolizumab pegol [ATC code: L04AB05], golimumab [ATC code: L04AB06], and ustekinumab [ATC code: L04AC05]). Patients were grouped and assessed separately, according to their index treatment line within the follow-up period. The use of PsA-related treatment during the characterization period was also evaluated. Naive-PsA patients were defined as those who had not received any PsA-related treatment (including both systemic nonbiologic and biologic therapies) before the year preceding the ID. Patients were defined "previously treated" if they had at least one prescription related to PsA in the characterization period.

The presence of concomitant autoimmune disease diagnoses (such as rheumatoid arthritis, ankylosing spondylitis, ulcerative colitis, and Crohn's disease) was evaluated during the characterization period. Comorbidities were measured using the Charlson Comorbidity Index $(\mathrm{CCI})^{12}$ that assigns a score to each concomitant disease identified through treatments and hospitalizations during the characterization period; the CCI score reflects a patient's overall health status. The methodology has been widely used as a way to compare disease severity in observational retrospective studies when data are unavailable.

Therapeutic pathways and drug utilization (in terms of switch and add-on) among patients enrolled, according to treatment type and baseline characteristics, were estimated during the follow-up period. A switch was defined as the presence of different therapy other than that administered at ID during the follow-up period or in the 60 days period following the end of the drug treatment line. An add-on was defined through the presence of prescription of another drug for the treatment of PsA before the end of follow-up period in addition to the index medication.

Measure of disease activity was based on C-reactive protein (CRP) value (from laboratory test outcomes database where available in LHU) at baseline and three different groups were created according to blood test results: high CRP, $>1 \mathrm{mg} / \mathrm{dL}$ $(10 \mathrm{mg} / \mathrm{L})$; medium, $>0.5 \mathrm{mg} / \mathrm{dL}(5 \mathrm{mg} / \mathrm{L})<\mathrm{CRP} \leq 1 \mathrm{mg} / \mathrm{dL}$ (10 mg/L); low, CRP $\leq 0.5 \mathrm{mg} / \mathrm{dL}(5 \mathrm{mg} / \mathrm{L})$.

\section{Cost analysis}

In order to estimate PsA management costs, the overall consumption of health care resources was evaluated during the follow-up period. Health care resource utilization and costs, based on resource consumption from ID, were assessed as an average per patient per year. Drug costs were evaluated using the Italian National Health System (NHS) purchase price. Hospitalization costs were determined using the diagnosis-related group (DRG) tariffs. DRG tariffs represent the reimbursement levels of the NHS to health care providers. The cost of instrumental and laboratory tests was defined according to the tariffs applied by regions. The cost analysis was conducted from the perspective of the NHS The currency reference used was Euro $(€)$.

\section{Statistical analysis}

Descriptive analyses are presented in this paper. Continuous variables were reported as mean (median and range as appropriate), whereas categorical variables were expressed as numbers and percentages. In cases where data were not issuable for data privacy - results referred to less than four patients, as potentially reconductable to single individuals "Codice in materia di protezione dei dati personali (Code for protection of personal data; D.Lgs. 196/2003)" ${ }^{\prime 13}$ - they have been reported as not issuable (NI).

\section{Results}

A total of 2,408 (prevalence 0.83 per 1,000) PsA patients were included in the analysis. Overall, $52 \%$ of patients were male, with a median age of 54 years; CCI was 1.1, and more frequent diseases were chronic obstructive pulmonary disease $17.2 \%$, diabetes $7.1 \%$, peptic ulcer disease $5.0 \%$, human immunodeficiency virus $1.7 \%$, and cancer $1.0 \%$.

Patients who were already treated with drugs indicated for PsA were $42.4 \%$ of cases ("previously treated patients"). Baseline characteristics by PsA treatment status are reported in Table 1.

At 1 year of follow-up, $73 \%$ of the patients received one systemic drug, while $22 \%$ received two systemic drugs (Figure 1).

More frequent csDMARDs (in green) at ID were methotrexate $(52 \%)$, sulphasalazine $(18 \%)$, cyclosporine 
Table I Baseline clinical characteristics

\begin{tabular}{|c|c|c|c|}
\hline & Total PsA patients & Naive patients & Previously treated patients \\
\hline $\mathrm{N}$ & 2,408 & $\mathrm{I}, 388$ & $\mathrm{I}, 020$ \\
\hline Prevalence per 1,000 & 0.83 & - & - \\
\hline Age, median (min-max) & $54(18-88)$ & $52(18-88)$ & $55(18-87)$ \\
\hline Male (n, \%) & $\mathrm{I}, 007(4 \mathrm{I} .8)$ & $552(39.8)$ & $455(44.6)$ \\
\hline Autoimmune disease $^{a}(n, \%)$ & $307(12.7)$ & $135(9.7)$ & $172(16.9)$ \\
\hline Charlson (mean) & 1.1 & 1.0 & I.I \\
\hline COPD (n, \%) & $415(17.2)$ & $248(17.9)$ & $167(16.4)$ \\
\hline Diabetes (n, \%) & $17 \mid(7.1)$ & $84(6.1)$ & $87(8.5)$ \\
\hline Peptic ulcer disease (n, \%) & $120(5.0)$ & $48(3.5)$ & $72(7.1)$ \\
\hline $\operatorname{HIV}(\mathrm{n}, \%)$ & $42(1.7)$ & $25(1.8)$ & $17(1.7)$ \\
\hline Cancer (n, \%) & $23(1.0)$ & $12(0.9)$ & II (I.I) \\
\hline Patients not previously treated $(\mathrm{n}, \%)$ & $1,020(42.4)$ & $0(0.0)$ & $1,020(100.0)$ \\
\hline Death at I year & $\mathrm{NI}$ & $\mathrm{NI}$ & $\mathrm{NI}$ \\
\hline
\end{tabular}

Note: aRheumatoid arthritis, ankylosing spondylitis, ulcerative colitis, and Crohn's disease.

Abbreviations: COPD, chronic obstructive pulmonary disease; HIV, human immunodeficiency virus; NI, not issuable; PsA, psoriatic arthritis.

(7\%), while more frequent bDMARDs (in gray) at ID were etanercept (5\%), adalimumab (4\%), and infliximab (1\%); the utilization of biologic agents was higher among patients with previous PsA treatment, showing a progression of the pathology (Figure 2). Percentage of patients included in the analysis in treatment with glucocorticosteroids was $8.4 \%$ (for naive patients: $53.1 \%$; for established patients: $42.0 \%$ ).

At 1 year of follow-up, treatment interruptions were observed among $31 \%$ of patients, while drug changes in $15 \%$, and add-on in $12 \%$; at 2 years of follow-up these percentages were $44 \%, 20 \%$, and $15 \%$, respectively, and at 3 years of follow-up, the percentages were $55 \%, 25 \%$, and $16 \%$, respectively, showing an increase in treatment changes. Switches occurred after a mean of 177 days (median 166 days). Among switchers, $77.3 \%$ of patients switched from one csDMARD to another, 5.1\% from one bDMARD to another bDMARD, $13.6 \%$ from csDMARD to bDMARD, and $4.0 \%$ from $b D M A R D$ to csDMARD; among patients who combined two different drugs, $57.8 \%$ combined two csDMARDs, while $42.2 \%$ combined one csDMARD with one bDMARD or one bDMARD with one csDMARD (Figure 3 ).
Baseline CRP levels ( \pm 6 months in relation to the ID) were analyzed. The analysis was performed on a subset of 432 patients for whom the laboratory values were available (Figure 4). Overall, a medium/high level of CRP at baseline was observed among more than half of the overall sample, with slight changes across subgroups in analysis. The average annual health care costs for the management of PsA patients, based on resource consumption from ID, were $€ 1,966.4$ and $€ 13,914$ per year for patients treated with csDMARD and bDMARD, respectively (Figure 5).

\section{Discussion}

PsA places a high burden on patient quality of life, productivity (indirect costs), and health care resources (direct costs). ${ }^{14-18}$ A literature review of 49 studies evaluating the burden of PsA revealed two studies that showed that the average annual direct and indirect cost associated with PsA ranged from $\approx \$ 8,367$ to $\$ 18,110 .{ }^{10}$ Hospitalizations accounted for almost $60 \%$ of direct costs, whereas disability and lost productivity accounted for the majority of indirect costs. Both direct and indirect costs increased with worsening

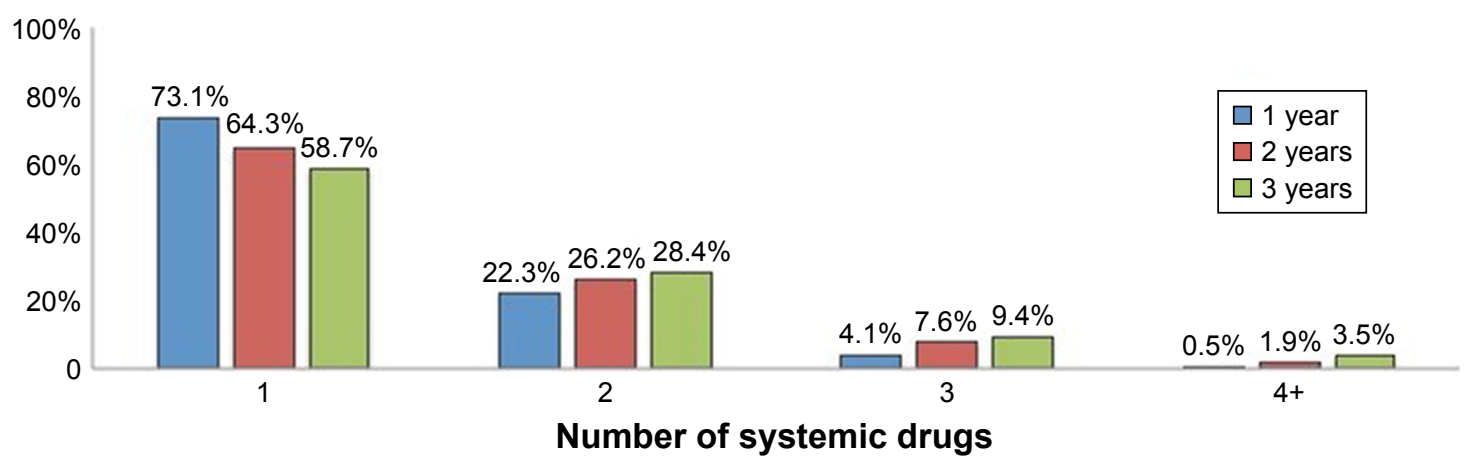

Figure I Drug utilization: number of systemic drugs used during the follow-up. 
A

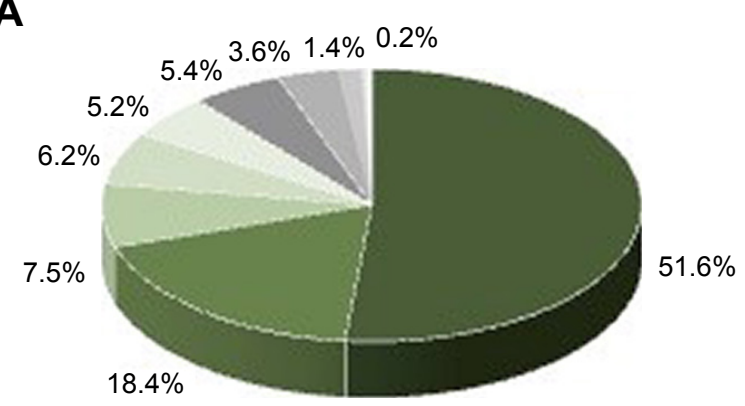

- Methotrexate

- Sulphasalazine

- Cyclosporine

Hydroxychloroquine

Leflunomide

= Etanercept

- Adalimumab

Infliximab

Ustekinumab

Others
B

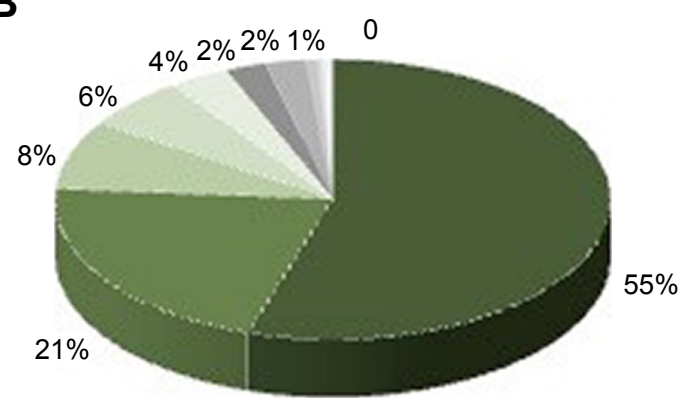

C

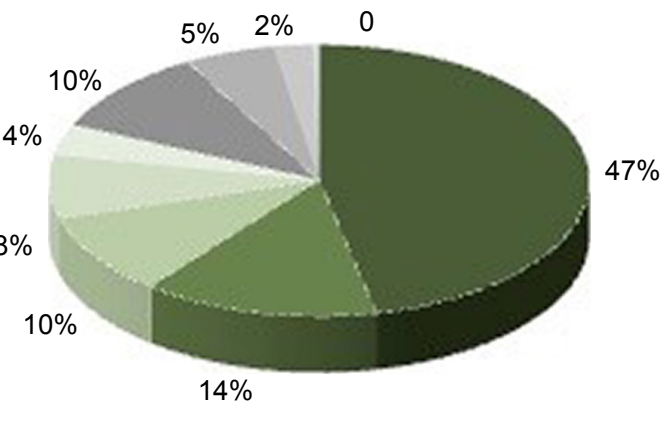

Figure 2 Drug use in the $(\mathbf{A})$ study population for $(\mathbf{B})$ naive cohort and $(\mathbf{C})$ previously treated cohort.

physical functioning and increased disease activity. Indirect costs accounted for $52 \%-72 \%$ of the total costs. ${ }^{10}$

A recent nationwide cohort study, using data from Danish registries, suggested that the health care costs for PsA patients increased from $<€ 2,000(\$ 2,137)$ annually 5 years prior to diagnosis to over $€ 5,000(\$ 5,344) /$ year at the time of diagnosis. ${ }^{15}$ The study also found that compared with the general population, PsA patients not only have increased health care costs but also have lower income, higher unemployment, greater risk for disability, and more comorbidities, both before and after their diagnosis. ${ }^{15}$

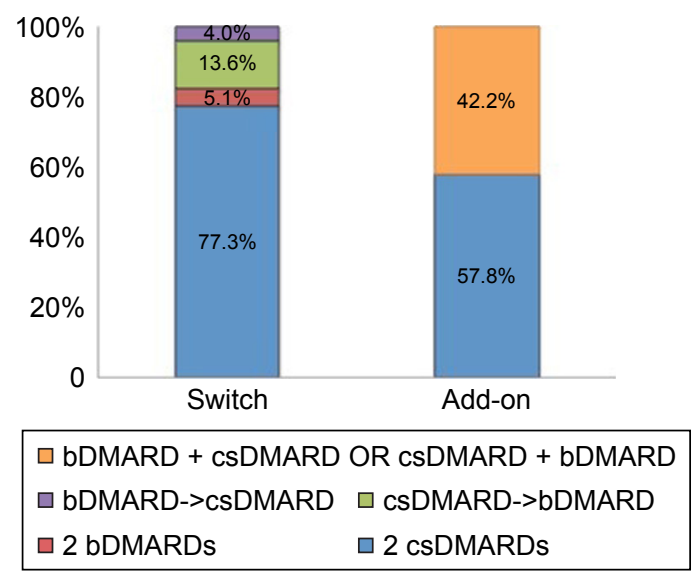

Figure 3 Drug utilization: systemic drug variations during follow-up. Abbreviations: bDMARD, biologic disease-modifying anti-rheumatic drug; csDMARD, conventional synthetic disease-modifying anti-rheumatic drug.
The therapeutic approach to PsA is broad-ranging, and includes a first step with conventional therapy (topical agents, corticosteroids, NSAIDs, and csDMARDs) and a second step with biologic (bDMARDs) therapies in refractory patients. The EULAR recommendations for the management of PsA with pharmacological therapies updated in 2015 highlighted that considering several therapeutic options available and insufficient information on differential efficacy and safety, treatment decisions in clinical practice remain challenging. ${ }^{8}$ Although the introduction of biologic agents seems to have led to a reduction in hospital costs for the management of PsA, the increase in direct and total cost identified in the studies of PsA showed the great economic impact of their incorporation into the therapeutic arsenal. ${ }^{19,20}$ The low number of studies focused on estimating the cost of PsA points to the need for and importance of future analyses of the cost of the disease. These studies should reflect clinical practice and provide useful and up-to-date information for decision-making in health care. Our findings confirm that, in an Italian real-world setting, costs are usually higher for patients treated with biologics.

As expressed by CRP levels, we found that the inflammatory burden in our PsA cohort was above the normal limits in a high proportion of patients receiving both csDMARD and bDMARD. Since a correlation between elevated levels of acute-phase proteins and the effectiveness of treatments have been noted, ${ }^{21}$ further research is required to assess both 


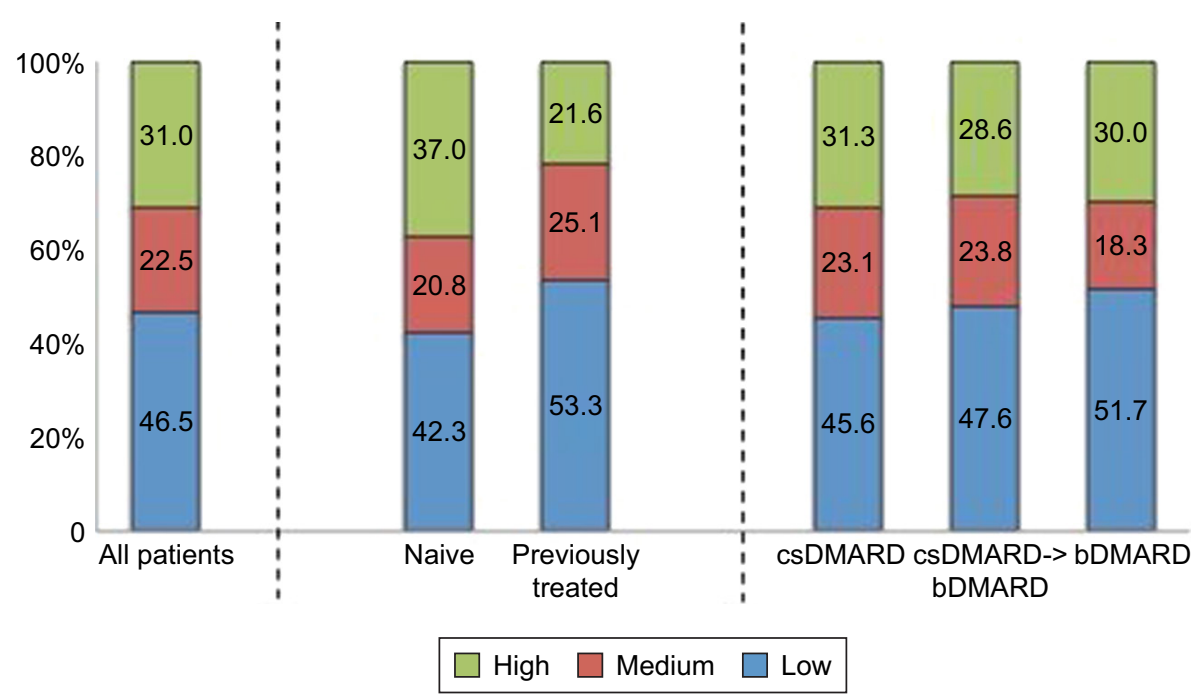

Figure 4 CRP at baseline.

Note: High CRP $>$ I mg/dL, medium $0.5 \mathrm{mg} / \mathrm{dL}<\mathrm{CRP} \leq \mathrm{I} \mathrm{mg} / \mathrm{dL}$, and low CRP $\leq 0.5 \mathrm{mg} / \mathrm{dL}$.

Abbreviations: bDMARD, biologic disease-modifying anti-rheumatic drug; CRP, C-reactive protein; csDMARD, conventional synthetic disease-modifying anti-rheumatic drug.

disease progression or disease remission in our patients and on relation of CRP levels with PsA cost.

Our data are also not dissimilar from those of other studies in the literature so far. ${ }^{22}$ The FRI0499 real-world study showed that the patterns and cost of treatment failure (defined as discontinuation and switching rates) in patients with PsA in a US managed care setting was high. In addition, follow-up costs for patients who switched were higher than those for patients who continued or discontinued their medication. $^{22}$

The present study has some limitations. Our cohort of patients reflected real clinical practice, and the results must be interpreted, taking into account the limitations related to the observational nature of the study, based on data collected through administrative and laboratory databases. A limitation was the lack of clinical information, such as data on comorbidities, the severity of the pathology, and other potential confounders that could have influenced our results. A proxy of the comorbidities was used. The results and conclusions of this study are limited to the population analyzed; further studies are necessary to confirm and enhance the generalizability of the findings.

The results of our "real life" study, being conducted in a limited number of Italian LHU, do not cover all the problems

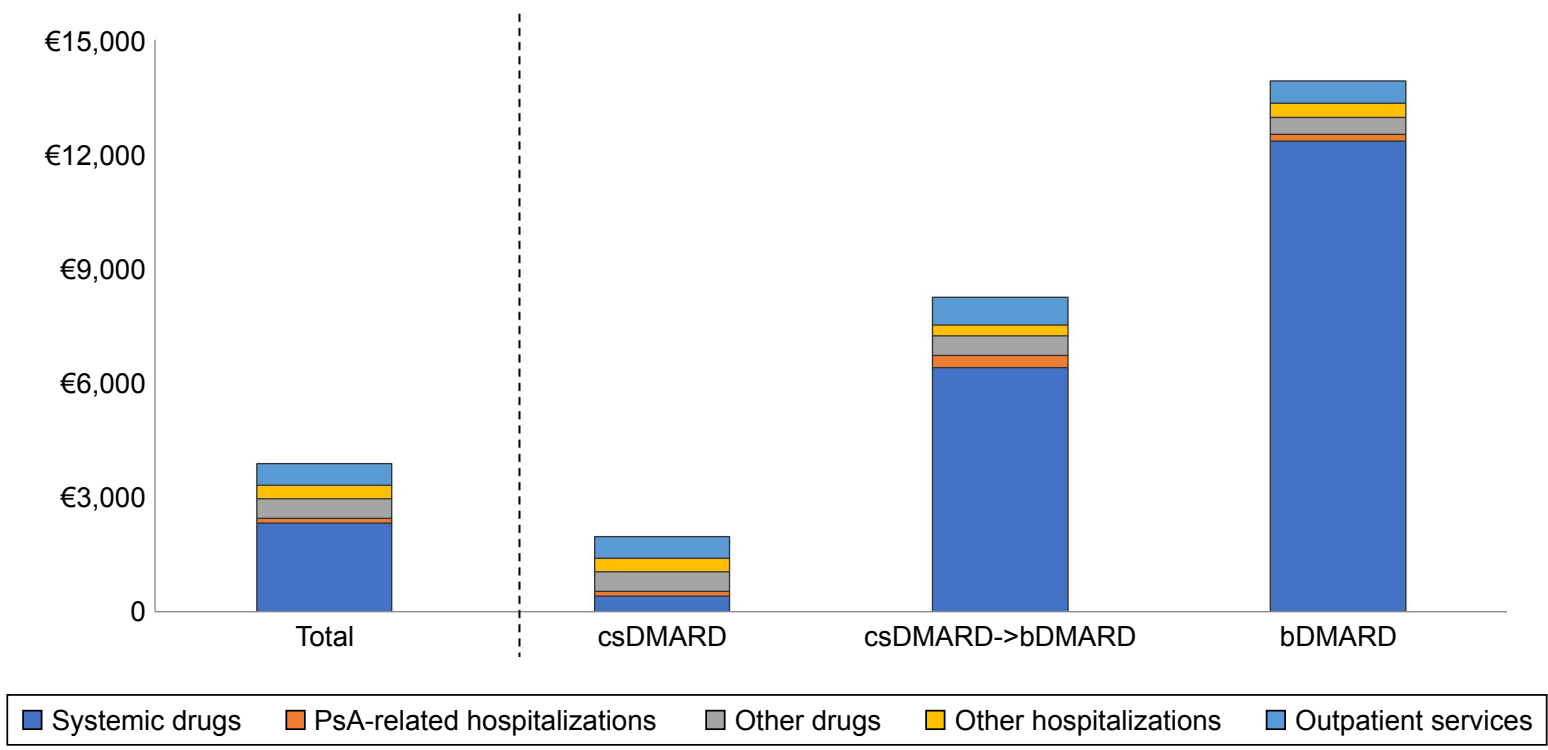

Figure 5 Annual per-patient cost of illness $(€)$.

Abbreviations: bDMARD, biologic disease-modifying anti-rheumatic drug; csDMARD, conventional synthetic disease-modifying anti-rheumatic drug; PsA, psoriatic arthritis. 
related to the treatments and costs associated with the management of patients with PsA, but rather give the possibility to optimize the resources used by NHS. A better knowledge of prescription scheme and economic burden of PsA could stimulate the rational development of health programs aimed at potentiating services for its management.

\section{Data sharing statement}

All data generated or analyzed during this study are included in this article.

\section{Acknowledgments}

CliCon S.r.1. is an independent company. The agreement sign by CliCon S.r.l. and Bristol-Myers Squibb does not create any entityship, joint venture, or any similar relationship between parties. Neither CliCon nor any of their representatives are employees of Bristol-Myers Squibb for any purpose. This study was funded by Bristol-Myers Squibb. The views expressed here are those of the authors and not necessarily those of the funders. The abstract of this paper was presented at the ISPOR 20th Annual European Congress as a poster presentation. The poster's abstract was published in "Poster Abstracts", Volume 20, Value in Health journal in November 2017, DOI: https://www.valueinhealthjournal.com/article/ S1098-3015(17)31114-2/fulltext.

This study was conducted on behalf of the following LHUs: D Ancona (Local Pharmaceutical Department, Barletta-Andria-Trani Local Health Authority, Italy); M Gambera (Local Pharmaceutical Service, Bergamo Local Health Authority, Bergamo, Italy); F Lena (Local Pharmaceutical Department, USL Tuscany South East Local Health Unit, Grosseto, Italy); E Nava (Department of Pharmacy, Torre del Greco Local Health Authority, Naples, Italy); S Palcic (Complex Pharmaceutical Assistance Unit, University Integrated Healthcare Unit of Trieste, Trieste, Italy); M Pisani (Primary Care Functional Unit, USL Tuscany South East Local Health Unit, Grosseto, Italy); A Pisterna (Local Pharmaceutical Service, Vercelli Local Health Authority, Italy); and A Vercellone (Department of Pharmacy, Torre del Greco Local Health Authority, Naples, Italy).

\section{Author contributions}

All authors contributed toward data analysis, drafting, and revising the paper, gave final approval of the version to be published, and agree to be accountable for all aspects of the work.

\section{Disclosure}

All the CliCon's authors (VP, LDE, DS, DA, SB, and FC) report no conflicts of interest in this work. EM, CT, and
FDS are employees of Bristol-Myers Squibb. They declare to not have any potential financial and/or personal conflict of interest in this work.

\section{References}

1. Dewing KA. Management of patients with psoriatic arthritis. Nurse Pract. 2015;40(4):40-46.

2. Liu JT, Yeh HM, Liu SY, Chen KT. Psoriatic arthritis: epidemiology, diagnosis, and treatment. World J Orthop. 2014;5(4):537.

3. Ogdie A, Weiss P. The epidemiology of psoriatic arthritis. Rheum Dis Clin North Am. 2015;41(4):545-568.

4. De Angelis R, Salaffi F, Grassi W. Prevalence of spondyloarthropathies in an Italian population sample: a regional community-based study. Scand J Rheumatol. 2007;36(1):14-21.

5. Ramiro S, Smolen JS, Landewé R, et al. Pharmacological treatment of psoriatic arthritis: a systematic literature review for the 2015 update of the EULAR recommendations for the management of psoriatic arthritis Ann Rheum Dis. 2016;75(3):490-498.

6. Coates LC, Kavanaugh A, Mease PJ, et al. Group for Research and Assessment of Psoriasis and Psoriatic Arthritis 2015 Treatment Recommendations for Psoriatic Arthritis: GRAPPA treatment recommendations for PsA. Arthritis Rheumatol. 2016;68(5):1060-1071.

7. Cantini F, Niccoli L, Nannini C, et al. Tailored first-line biologic therapy in patients with rheumatoid arthritis, spondyloarthritis, and psoriatic arthritis. Semin Arthritis Rheum. 2016;45(5):519-532.

8. Gossec L, Smolen JS, Ramiro S, et al. European League Against Rheumatism (EULAR) recommendations for the management of psoriatic arthritis with pharmacological therapies: 2015 update. Ann Rheum Dis. 2016;75(3):499-510.

9. Gossec L, Smolen JS, Gaujoux-Viala C, et al. European League Against Rheumatism recommendations for the management of psoriatic arthritis with pharmacological therapies. Ann Rheum Dis. 2012;71(1):4-12.

10. Lee S, Mendelsohn A, Sarnes E. The burden of psoriatic arthritis: a literature review from a global health systems perspective. $P$ T. 2010;35: 680-689.

11. Agenzia Italiana del Farmaco (AIFA). Guideline for the classification and conduction of the observational studies on medicines; 2010. Available from: https://www.agenziafarmaco.gov.it/ricclin/sites/ default/files/files_wysiwyg/files/CIRCULARS/Circular\%2031st $\% 20$ May\%202010.pdf

12. Gonnella JS, Louis DZ, Gozum MV, Callahan CA, Barnes CA. Disease Staging Clinical and Coded Criteria (Version 5.26). Ann Arbor, MI, USA: Thomson Medstat; 2010.

13. Opinion 05/2014 on Privacy and Data Protection. Available from: http://ec.europa.eu/justice/data-protection/article-29/documentation/ opinion-recommendation/files/2014/wp216_it.pdf

14. Olivieri I, de Portu S, Salvarani C, et al. The psoriatic arthritis cost evaluation study: a cost-of-illness study on tumour necrosis factor inhibitors in psoriatic arthritis patients with inadequate response to conventional therapy. Rheumatology. 2008;47(11): $1664-1670$.

15. Kristensen LE, Jørgensen TS, Christensen R, et al. Societal costs and patients' experience of health inequities before and after diagnosis of psoriatic arthritis: a Danish cohort study. Ann Rheum Dis. 2017;76(9): 1495-1501.

16. Barra L, Pope JE, Payne M. Real-world anti-tumor necrosis factor treatment in rheumatoid arthritis, psoriatic arthritis, and ankylosing spondylitis: cost-effectiveness based on number needed to treat to improve health assessment questionnaire. $J$ Rheumatol. 2009;36(7): $1421-1428$.

17. Kavanaugh A, Helliwell P, Ritchlin CT. Psoriatic arthritis and burden of disease: patient perspectives from the population-based multinational assessment of psoriasis and psoriatic arthritis (MAPP) survey. Rheumatol Ther. 2016;3(1):91-102.

18. Tillett $\mathrm{W}$, de-Vries $\mathrm{C}, \mathrm{McHugh}$ NJ. Work disability in psoriatic arthritis: a systematic review. Rheumatology. 2012;51(2):275-283. 
19. Burgos-Pol R, Martínez-Sesmero JM, Ventura-Cerdá JM, Elías I, Caloto MT, Casado MÁ. The cost of psoriasis and psoriatic arthritis in 5 European countries: a systematic review. Actas Dermosifiliogr. 2016;107(7):577-590.

20. Lubrano E, Spadaro A. Pharmacoeconomic burden in the treatment of psoriatic arthritis: from systematic reviews to real clinical practice studies. BMC Musculoskelet Disord. 2014;15:25.
21. Chandran V, Gladman DD. Update on biomarkers in psoriatic arthritis. Curr Rheumatol Rep. 2010;12(4):288-294.

22. Price K. FRI0499 Real-world study on the patterns and cost of treatment failure in patients with psoriatic arthritis using us claims data in 677.2-677. BMJ Publishing Group Ltd and European League Against Rheumatism; 2017;76(Suppl 2):677.2-677.

\section{Publish your work in this journal}

Patient Preference and Adherence is an international, peer-reviewed, open access journal that focuses on the growing importance of patient preference and adherence throughout the therapeutic continuum. Patient satisfaction, acceptability, quality of life, compliance, persistence and their role in developing new therapeutic modalities and compounds to optimize clinical outcomes for existing disease states are major areas of interest for the journal. This journal has been accepted for indexing on PubMed Central. The manuscript management system is completely online and includes a very quick and fair peer-review system, which is all easy to use. Visit http://www. dovepress.com/testimonials.php to read real quotes from published authors.

Submit your manuscript here: http://www.dovepress.com/patient-preference-and-adherence-journal 